\title{
ВАРИАБЕЛЬНОСТЬ ГЛИКЕМИИ ПРИ САХАРНОМ ДИАБЕТЕ 2 ТИПА У ПАЦИЕНТОВ С НАЛИЧИЕМ КОГНИТИВНЫХ НАРУШЕНИЙ
}

\author{
Лейман О.П., Самойлова Ю.Г., Милованова Т.А., Матвеева М.В.
}

ФГБОУ «Сибирский государственный медицинский университет» Минздрава Российской Федерации

ЦЕЛь: проанализировать наличие и выраженность вариабельности гликемии у пациентов с сахарным диабетом 2 типа и наличием когнитивных нарушений в возрасте до 65 лет без острого нарушения мозгового кровообращения в анамнезе.

МАТЕРИАЛЫ И МЕТОДЫ: 70 пациентов с сахарным диабетом 2 типа, средний возраст 58,3土2,1, которым в рутинной амбулаторной практике и в условиях дневного стационара было проведено мониторирование уровня гликемии. Все пациенты были разделены на 2 группы: 1 группа (основная) - с наличием когнитивных нарушений (n=36), 2 группа (контрольная) - с нормальными когнитивными функциями (n=34). Всем пациентам проведен скрининг когнитивных функций с помощью Монреальской шкалы (МоСа теста). Для диагностики колебаний уровня глюкозы проведено непрерывное мониторирование гликемии с использованием прибора iPro-2 (Medtronic, США) с расчетом коэффициентов: среднее значение гликемии (MEAN), стандартное отклонение (SD), средняя амплитуда колебаний гликемии (MAGE), индекс длительного повышения гликемии (CONGA), индекс лабильности гликемии (LI), индекс риска гипогликемии (LBGI), индекс риска гипергликемии (HBGI), среднечасовая скорость изменения гликемии (MAG). Статистический анализ результатов будет проведен с помощью программы IBM SPSS Statistics 17.0.

PЕЗУЛЬТАТЫ: в результате исследования выявлено, что у пациентов с сахарным диабетом 2 типа 1 группы среди когнитивных нарушений преобладали нарушение конструктивного праксиса, памяти и внимания. Зарегистрировали значимую разницу в показателях MEAN, SD, CONGA, Gindex, LBGI, HBGl, MAGE, Mvalue и MAG. При проведении корреляционного анализа выявлена связь когнитивных нарушений с уровнем $\mathrm{HbA}_{1 c^{\prime}}$ а также параметрами вариабельности MEAN, SD, CONGA, Gindex, LBGI, HBGI, MAGE, $M$ value, MAG.

При проведении корреляционного анализа показано, что на когнитивные функции в целом влияет уровень $\mathrm{HbA}_{1 c}\left(\mathrm{X}^{2}=-0,450, \mathrm{p}=0,014\right)$, а также параметры вариабельности MEAN $\left(\mathrm{X}^{2}=-0,584, \mathrm{p}=0,001\right)$, $\operatorname{SD}\left(x^{2}=0,022, p=0,022\right), \operatorname{CONGA}\left(x^{2}=-0,853, p=0,001\right)$, Gindex $\left(x^{2}=-0,504, p=0,005\right), \operatorname{LBGl}\left(x^{2}=-0,451, p=0,014\right)$, HBGI $\left(x^{2}=-0,053, p=0,003\right)$, MAGE $\left(x^{2}=-0,480, p=0,008\right)$, Mvalue $\left(x^{2}=-0,593, p=0,001\right)$, MAG $\left(x^{2}=-0,573, p=0,001\right)$. На конструктивный праксис в задание альтернирующий путь оказывает наибольшее влияние параметр CONGA $\left(x^{2}=--0,502, p=0,006\right), \operatorname{MAGE}\left(x^{2}=--0,555, p=0,002\right)$, а в задание часы - MEAN $\left(x^{2}=--0,379, p=0,043\right)$, LI $\left(x^{2}=-0,471, p=0,010\right)$, Gindex $\left(x^{2}=--0,497, p=0,006\right)$, LBGI $\left(x^{2}=--0,477, p=0,009\right)$, HBGl $\left(x^{2}=--0,384, p=0,040\right)$, MAGE ( $\left.x^{2}=--0,386, p=0,038\right)$, Mvalue $\left(x^{2}=--0,546, p=0,002\right)$, MAG $\left(x^{2}=--0,505, p=0,005\right)$. Для снижения памяти наибольшее значение имеет показатель MEAN ( $\left.X^{2}=--0,455, p=0,013\right)$. Нарушение выполнения задание на повторение фразы зависит от уровня $\mathrm{HbA}_{1 c}\left(X^{2}=--0,390, p=0,036\right), \mathrm{LI}\left(X^{2}=--0,463, p=0,011\right), \mathrm{LBGl}\left(X^{2}=--0,604\right.$, $p=0,001)$, MAGE $\left(x^{2}=--0,402, p=0,031\right)$, Mvalue $\left(x^{2}=-0,483, p=0,008\right)$, MAG $\left(x^{2}=--0,501, p=0,006\right)$.

ВЫВОДЫ: зарегистрирована связь вариабельности гликемии и когнитивных нарушений у пациентов с сахарным диабетом 2 типа 